\title{
Outcome of locking plate fixation in cases of comminuted proximal ulna fractures
}

\author{
Sanjeev Kumar Singhal ${ }^{1, *}$, V K Sahni ${ }^{2}$, Manoj Garg ${ }^{3}$, Anand Gupta ${ }^{4}$ \\ ${ }^{\mathbf{1 , 2 , 3}}$ Senior Consultant, ${ }^{4}$ Resident Doctor, Dept. of Orthopaedics, Maharaja Agrasen Hospital, Delhi, India \\ *Corresponding Author: Sanjeev Kumar Singhal \\ Email: sanjeevsinghal@yahoo.com
}

\begin{abstract}
Introduction: The purpose of this study was to report the clinical and radiological outcome following treatment with locking plate fixation in patients of comminuted fractures of the proximal ulna including olecranon and Monteggia fractures.

Materials and Methods: Between 2015 and 2017, thirty patients with comminuted fractures of the proximal ulna including 21 cases of fracture olecranon and 9 cases of Monteggia fractures were managed with locking plate fixation. The patients were followed for clinical and radiological examination for minimum 6 months post operatively. Validated patient-oriented assessment scores involving the Mayo Elbow Performance Score (MEPS) and postoperative range of motion and patient's satisfaction were evaluated.

Results: The mean arc of elbow motion in 21 cases of olecranon fractures was $108^{\circ}$ and mean MEPS was 89.3 with $57 \%$ excellent and $43 \%$ good results. While in 9 cases of Monteggia fractures mean arc of motion was $108^{\circ}$ and mean MEPS were 93.8 with $89 \%$ excellent and $11 \%$ good results. All patients achieved fracture union by 24 weeks. Mean duration of fracture union for olecranon fractures was 16.1 weeks and for Monteggia fractures 14.3 weeks.

Conclusion: Locking plate is an effective fixation method for fractures of the proximal ulna allowing reliable stability for early elbow motion. The functional results are comparable with formerly described plating systems. The good radiological outcomes highlight the usefulness of plate fixation in cases of proximal ulna fractures, provided optimal plate positioning achieved.
\end{abstract}

Keywords: Proximal ulna, Comminution, Locking plate, Olecranon fracture, Monteggia, Internal fixation.

\section{Introduction}

Proximal one third ulna fractures are common adult injuries around the elbow, which comprise broad spectrum of injuries including olecranon fractures and Monteggia fractures. The majority of olecranon fractures are transverse fractures with no or limited comminution (up to three fragments). These can be treated with the classic tension band fixation using two Kirschner wires and a tension band. ${ }^{1,2}$ Complex fractures of the proximal ulna with severe comminution have become more frequent these days. However, these fractures still remain a treatment challenge for trauma surgeons. The treatment goals are to obtain anatomic reduction that remains stable over time, bone healing and functional recovery. To allow early functional mobilization and to prevent stiffness of the elbow joint, the fracture fixation has to provide secure stability. ${ }^{3,4}$ Especially for severely comminuted proximal ulna fractures including comminuted olecranon fractures, fractures coronoid process and Monteggia fractures, plate fixation has evolved as gold standard treatment. ${ }^{1,5,6}$ Different studies reported promising results for dorsal, contoured plating of complex proximal ulnar fractures. ${ }^{7-9} \mathrm{In}$ more recent times, the use of locked plating is being advocated more and more frequently. ${ }^{10,11}$ In these cases tension band wiring may lead to collapse of the fragments with shortening of the articular surface of the olecranon, incongruity of the joint, impingement, loss of movement, and degenerative osteoarthritis. ${ }^{1,2,7}$ The purpose of contoured dorsal plate fixation in these fractures is to simulate the function of a tension band after repositioning of the fragments. Dorsal plate also acts as a buttress to prevent fracture flexion in cases of deficient anterior cortex. ${ }^{6}$ Aim of this study was to observe clinical and radiological outcome of locking plate fixation in cases of comminuted proximal ulna fractures.

\section{Materials and Methods}

This prospective study was hospital based and was conducted at a tertiary care hospital at New Delhi, India. Patients were selected from those who attended the emergency and outpatient department. Written informed consent was obtained from all the patients or their family members for participation in the study. For this study we recruited thirty patients of comminuted fracture proximal ulna including fracture olecranon and Monteggia fracture, who presented to our hospital between July 2015 to March 2017 and were followed for minimum six months. Patients were enrolled based on inclusion and exclusion criteria. Patients of more than 18 years of age with comminuted proximal one third ulna fracture of less than one month old were included in the study. Patients with additional ipsilateral upper extremity injury or who were unfit for surgery were excluded.

On admission, demographic data was recorded and thorough history and clinical examination was done. Neurovascular status and radiological assessment of the fractured limb was done. Patients were investigated further depending on the general condition and comorbidity of the patient and the routine pre-operative protocol was followed as per our hospital guidelines. Preoperatively the decision of the exact modality of surgery and fixation was made. Pre-operative surgical 
antibiotic prophylaxis was given within one hour of surgery. Patients were given general or regional anaesthesia. Position of the patient was lateral decubitus with arm over padded bar allowing elbow flexion. Image intensifier was kept for intra-operative imaging if required. We used direct posterior midline approach for olecranon fractures while for Monteggia fracture we used Speed and Boyd' approach.

Postoperatively antibiotics were given as per hospital protocol. Analgesics and other supportive management were given according to the patient need. The patients were discharged according to the overall well-being of the patient, preferably on third or fourth day with medications convenient to be taken at home. Range of motion exercises of the shoulder and elbow was begun within two weeks. Post operatively patients underwent thorough clinical evaluation including assessment of functional status using Mayo elbow performance score along with radiological evaluation.

Patients were followed at 2 weeks (stitch removal), 6 weeks, 3 months and at 6 months of surgery. During follow up, course of fracture healing was documented radiologically (with minimum of 6 weeks between successive radiographs). Fracture union was defined as union of three out of four cortices on two orthogonal radiographs. Patients were assessed for functional status using Mayo Elbow performance score and analysis of any complications observed in terms of loss of reduction, infection, problems of union and implant failure. Delayed union and non-union were defined as failure to fracture union at 16 weeks and 6 month postoperative period, respectively.

\section{Results}

In our prospective study, 21 patients with comminuted olecranon fracture and 9 patients with Monteggia fracture were treated using locking plate fixation during the period of July 2015 to March 2017. Clinical and radiological evaluation of the patients was done with minimum 6 months follow up.

Olecranon Fractures: More than two third $(n=16)$ of total 21 patients of olecranon fractures were male and left side was more common to involved than right side. Fall on the ground and road traffic accident were the major cause of injury, almost equally contributed to these fractures. Out of twenty one patients of olecranon fractures, thirteen patients belonged to Mayo type II A, four each of Mayo Type II B and Mayo type IIIA olecranon fracture (Table 1).

All twenty one patients achieved fracture union in 6 months follow up period. Out of 21 patients, 15 (71.4\%) had fracture union by 16 weeks. 5 patients (23.8\%) achieved fracture union by 20 weeks while 1 patient $(4.8 \%)$ took 22 weeks. Mean duration of fracture union of olecranon fractures was 16 weeks. No patient had any implant related complication like implant failure, implant breakage or loosening. No case of any infection reported. Six patients had delayed union. No case of non-union was seen.

Assessment of range of motion at elbow joint on follow ups yielded progressively improving results with time. At 6 months follow up Mean Mayo elbow performance score was 89.3 (Table 2).

Monteggia Fracture: Out of 9 patients, almost half $(n=5)$ were male and rest were female. Left and right side affected almost equally. Road traffic accident was the major cause of injury affecting 8 out of total 9 patients. Two third of the patients were of type I while rest one third were type II of Bado classification (Table $3)$. All nine patients had fracture union by 16 weeks. Mean duration of fracture union was 14 weeks. No complication reported in any case.

In cases of Monteggia fracture also range of motion at elbow joint on follow ups yielded progressively improving results with time. Mean Mayo elbow performance score at 6 months follow up was 93.8 (Table 4). All articular congruent extraarticular fractures (Monteggia fractures) showed better arc of motion and Mayo elbow performance score than articular congruent intrarticular fractures (Olecranon fractures).

Table 1: Demographic details of Olecranon fracture patients

\begin{tabular}{|l|c|c|}
\hline Age Groups (Years) & $\begin{array}{c}\text { Number of } \\
\text { Patients }\end{array}$ & Percentage \\
\hline $21-30$ & 4 & $19 \%$ \\
\hline $31-40$ & 6 & $28.6 \%$ \\
\hline $41-50$ & 2 & $9.5 \%$ \\
\hline $51-60$ & 5 & $23.8 \%$ \\
\hline$>60$ & 4 & $19 \%$ \\
\hline Sex & & \\
\hline Male & 16 & $76.2 \%$ \\
\hline Female & 5 & $23.8 \%$ \\
\hline Side Involved & & \\
\hline Right & 8 & $38 \%$ \\
\hline Left & 13 & $62 \%$ \\
\hline Mayo Classification & & \\
\hline IIA & 13 & $62 \%$ \\
\hline IIB & 4 & $19 \%$ \\
\hline IIIA & 4 & $19 \%$ \\
\hline
\end{tabular}

Table 2: Post operative range of motion in cases of olecranon fractures

\begin{tabular}{|l|c|c|c|}
\hline Range of Motion & 6 weeks & $\begin{array}{c}\mathbf{3} \\
\text { months }\end{array}$ & $\begin{array}{c}\mathbf{6} \\
\text { months }\end{array}$ \\
\hline $\begin{array}{l}\text { Mean Flexion } \\
\text { Extension Arc }\end{array}$ & $77.9^{\circ}$ & $94.5^{\circ}$ & $108.1^{\circ}$ \\
\hline Mean Flexion & $17.4^{\circ}$ to & $12^{\circ}$ to & $10^{\circ}$ to \\
Extension Range & $95.3^{\circ}$ & $106.6^{\circ}$ & $118.3^{\circ}$ \\
\hline Mean Supination & $64^{\circ}$ & $69.04^{\circ}$ & $73.1^{\circ}$ \\
\hline Mean Pronation & $61.9^{\circ}$ & $66.2^{\circ}$ & $70.5^{\circ}$ \\
\hline $\begin{array}{l}\text { Mean Mayo Elbow } \\
\text { Performance Score }\end{array}$ & 81.2 & 85.5 & 89.3 \\
\hline
\end{tabular}


Table 3: Demographic details of Monteggia fracture dislocation patients

\begin{tabular}{|l|c|c|}
\hline Age Groups (Years) & $\begin{array}{c}\text { Number of } \\
\text { Patients }\end{array}$ & Percentage \\
\hline $21-30$ & 4 & $44 \%$ \\
\hline $31-40$ & 3 & $33 \%$ \\
\hline $41-50$ & 1 & $11 \%$ \\
\hline $51-60$ & 1 & $11 \%$ \\
\hline Sex & & \\
\hline Male & 5 & $56 \%$ \\
\hline Female & 4 & $44 \%$ \\
\hline Side Involved & & \\
\hline Right & 4 & $44 \%$ \\
\hline Left & 5 & $56 \%$ \\
\hline Bado Classification & & \\
\hline I & 6 & 67 \\
\hline II & 3 & 33 \\
\hline
\end{tabular}

Table 4: Post operative range of motion in cases of Monteggia fracture dislocation

\begin{tabular}{|l|c|c|c|}
\hline Range of Motion & 6 weeks & $\begin{array}{c}\mathbf{3} \\
\text { months }\end{array}$ & 6 months \\
\hline $\begin{array}{l}\text { Mean Flexion } \\
\text { Extension Arc }\end{array}$ & $79.4^{\circ}$ & $95.6^{\circ}$ & $108.9^{\circ}$ \\
\hline Mean Flexion & $15^{\circ}$ to & $10^{\circ}$ to & $8.9^{\circ}$ to \\
Extension Range & $94.4^{\circ}$ & $105.6^{\circ}$ & $117.8^{\circ}$ \\
\hline Mean Supination & $65.6^{\circ}$ & $70.6^{\circ}$ & $75.6^{\circ}$ \\
\hline Mean Pronation & $62.2^{\circ}$ & $67.8^{\circ}$ & $71.1^{\circ}$ \\
\hline $\begin{array}{l}\text { Mean Mayo Elbow } \\
\text { Performance Score }\end{array}$ & 83.3 & 88.3 & 93.8 \\
\hline
\end{tabular}

Case 1: Mayo type II B olecranon fracture
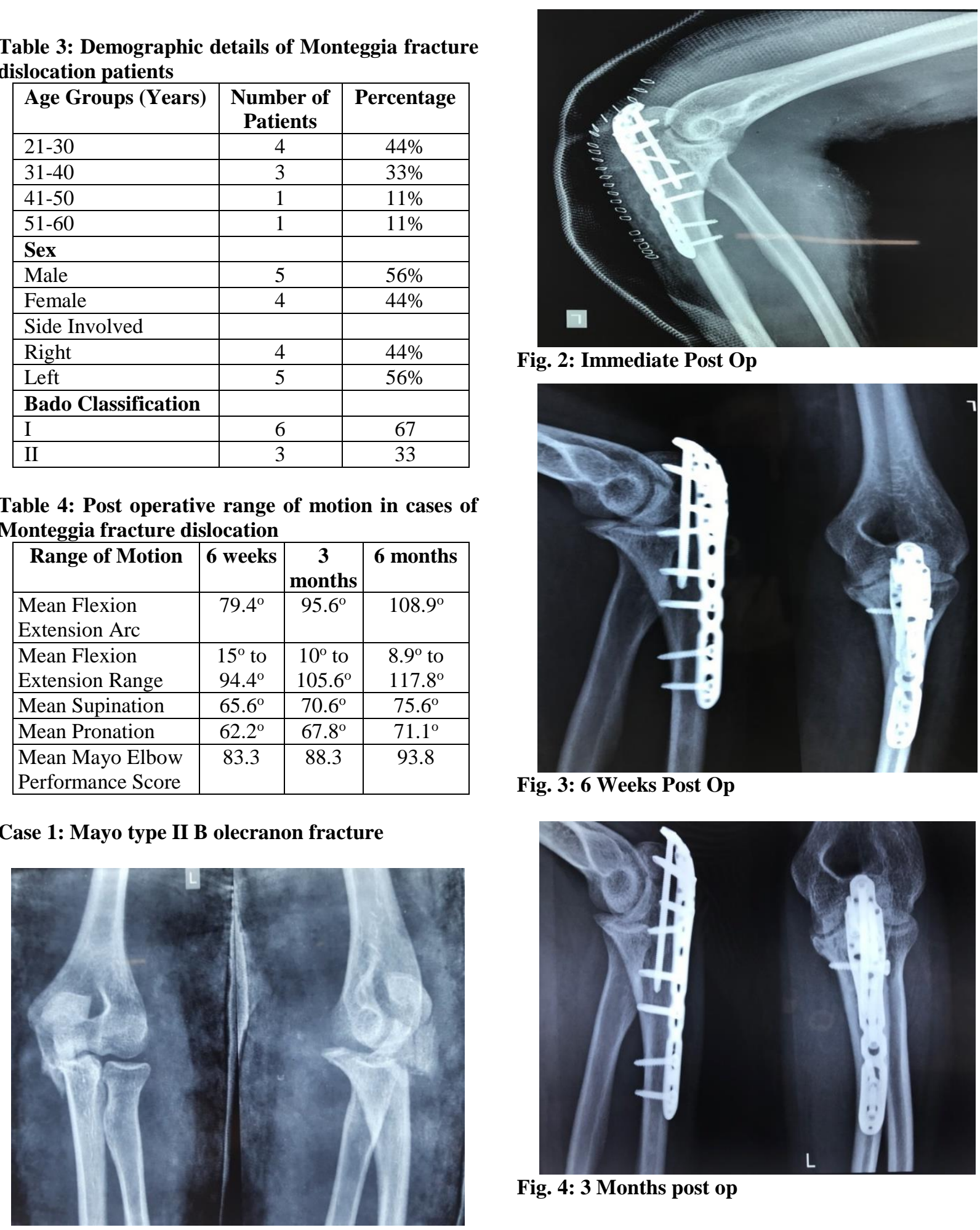

Fig. 2: Immediate Post Op

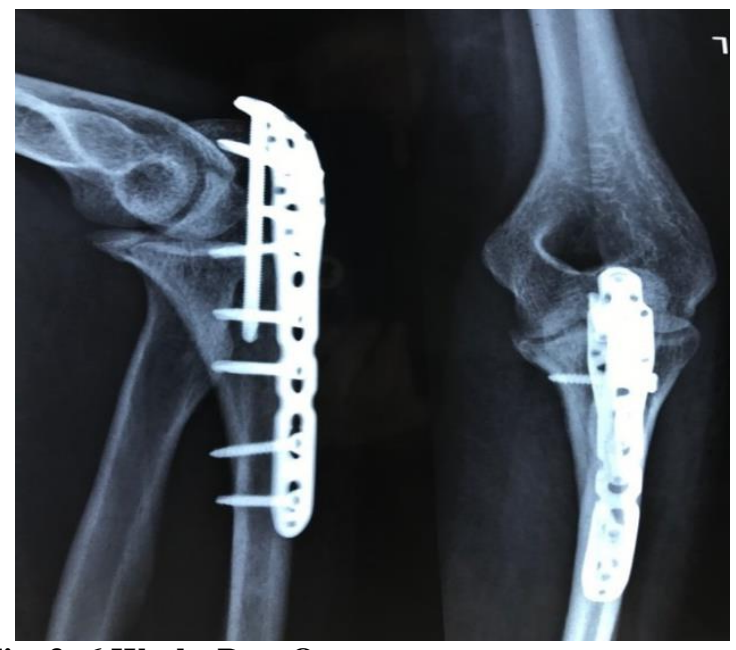

Fig. 3: 6 Weeks Post Op

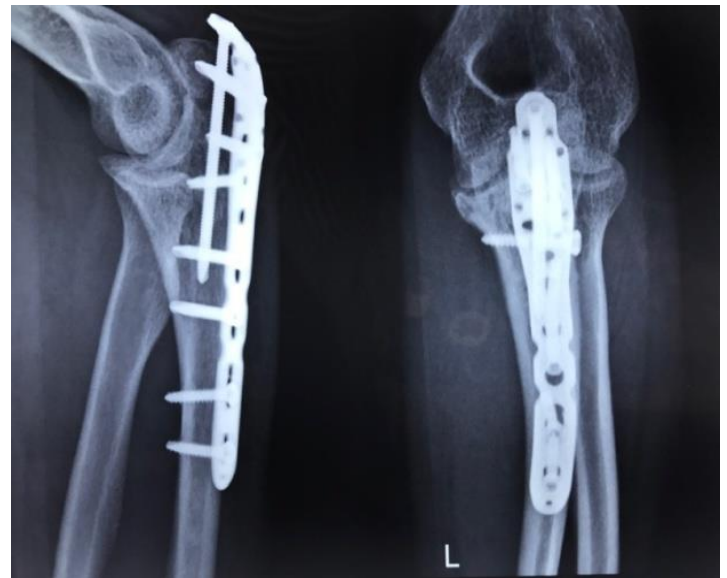

Fig. 4: 3 Months post op

Fig. 1: Pre Op 


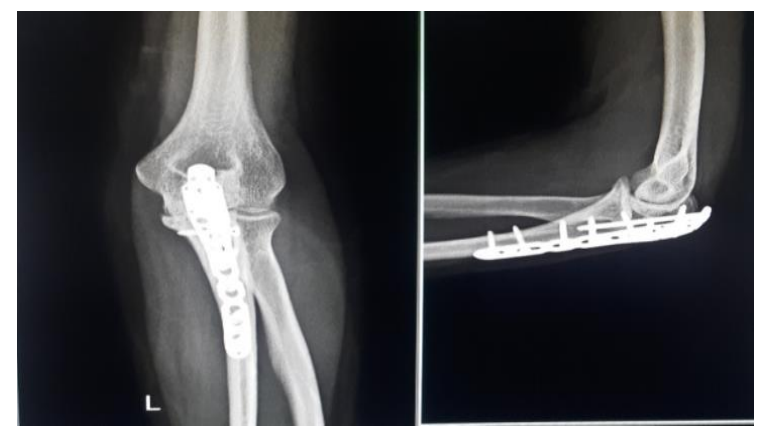

Fig. 5: 6 Months post op

\section{Case 2: Mayo type III A olecranon fracture}

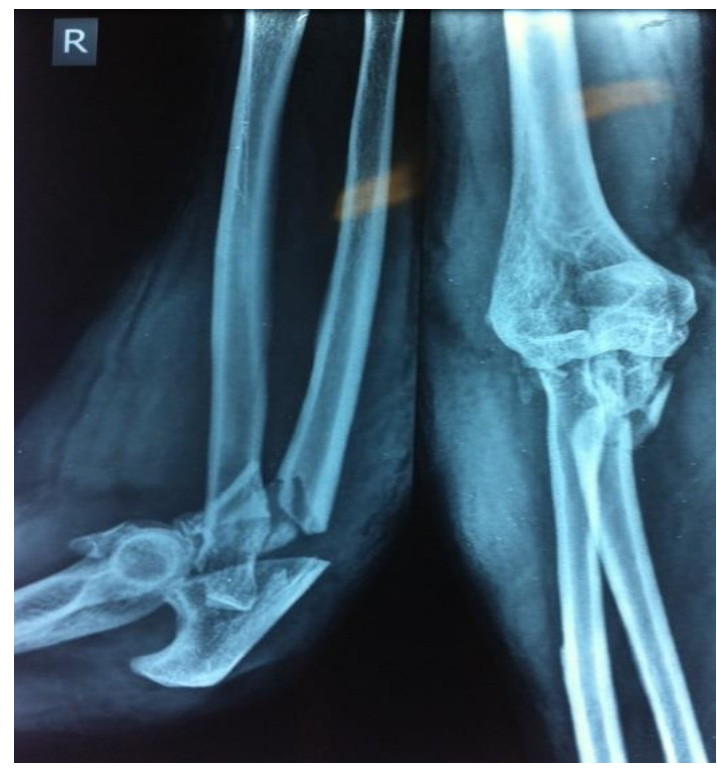

Fig. 6: Pre op

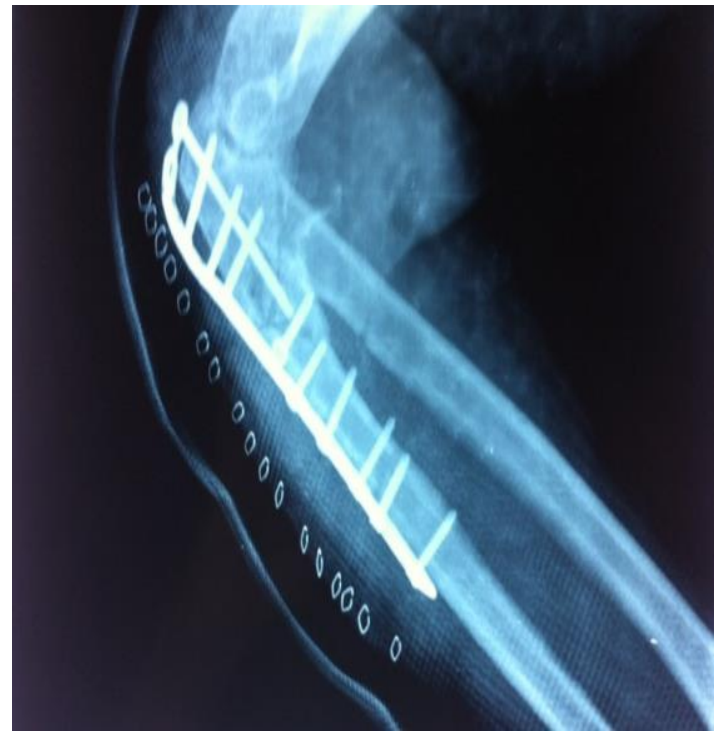

Fig. 7: Immediate post op
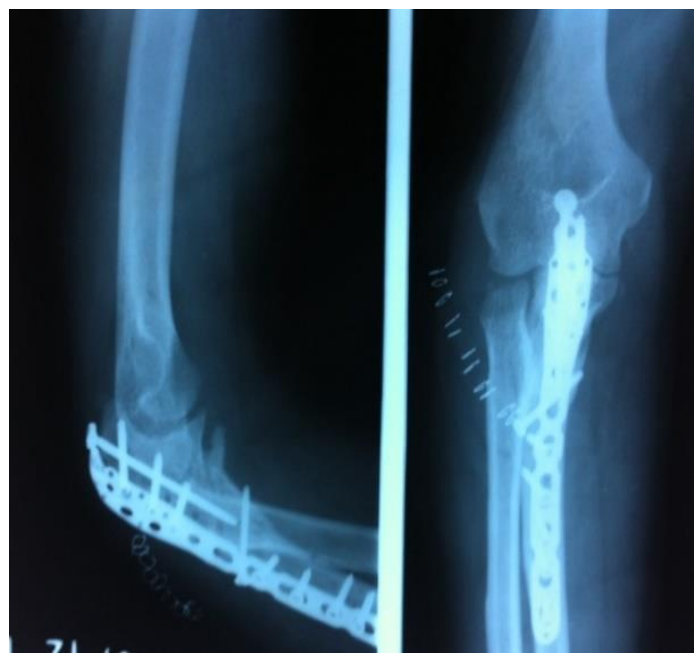

Fig. 8: Immediate post op
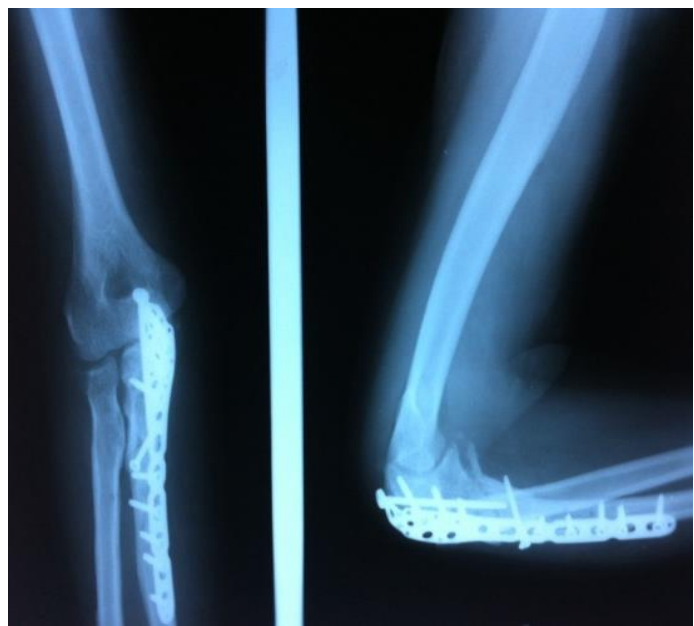

Fig. 9: 6 Months post op

\section{Discussion}

Proximal one third ulna fractures are common adult injuries that account for approximately $10 \%$ of fractures around the elbow including olecranon and Monteggia fractures. The elbow plays a critical part in the normal arm function. Comminuted fractures of proximal ulna threaten the integrity of both the elbow and forearm joints. The treatment goals are to maintain a stable and anatomic reduction, realign the longitudinal axis of the proximal ulna, and enable immediate rehabilitation.

Open reduction and stable internal fixation with the goal of anatomical reduction of the articular surface is the gold standard for olecranon fracture treatment. Non comminuted olecranon fractures can be treated by tension band wiring or plating. In cases of comminute fractures of proximal ulna it has many pitfalls like loss of fixation, prominence of hardware, impingement and synostosis. Use of locking plate avoids these complications and can also be used in comminuted as well as non-comminuted fractures. It also provides structural stability, resists ulnar angulation, and restores ulna length. In addition, plate fixation lowers the risk of 
fatigue failure caused by extreme bending stresses. Operative treatment by plating has been shown to provide more predictable alignment and immediate fracture stability, allowing early elbow mobilization.

We treated twenty one patients with olecranon fractures and nine patients of Monteggia fractures by locking plate fixation. The results obtained in our study were favorable. Mean age of the patients in our study was 45.8 years for olecranon fractures and 33 years in cases of Monteggia fracture with overall male predominance. Fall on ground was the more common mode of injury in cases of olecranon fractures while Monteggia fractures were mainly due to road traffic accident.

Our study included 13 Mayo type IIA and 4 each of type IIB and type IIIA olecranon fractures. While out of 9 patients with Monteggia fracture 6 belong to Bado Type I and rest 3 belong of Type II. ${ }^{13}$ All fractures in our study had united by 24 weeks, both clinically and radiologically. Mean duration of fracture union for olecranon fractures was 16.1 weeks and for Monteggia fracture was 14.3 weeks which is comparable to study done by Wang et al for olecranon fractures and by Siebenlist et al in cases of Monteggia fracture dislocation. ${ }^{14,15}$

For Olecranon fractures, mean arc of elbow motion was $108.1^{\circ}$ with range of motion from $10.23^{\circ}$ to $118.33^{\circ}$ and for Monteggia fracture mean arc of elbow motion was $108.9^{\circ}$ with range of motion from $8.9^{\circ}$ to $117.8^{\circ}$. Out of thirty patients, four patients had extension lag of $5^{\circ}$, twenty five patients had extension lag of $10^{\circ}$ and one patient had extension lag of $25^{\circ}$, nearly same observations were reported by Niglis et al and $\mathrm{Li}$ et al in their studies. ${ }^{16} \mathrm{We}$ observed that mean elbow supination and pronation was $73.1^{\circ}$ and $70.5^{\circ}$ respectively in cases of olecranon fractures and in cases of Monteggia fracture mean elbow supination and pronation was $75.6^{\circ}$ and $71.1^{\circ}$. Study done by Wang et al and Kloen et al reported almost same results. ${ }^{14,17}$

Mayo elbow performance score (MEPS) was used to know the functional outcome, as it depicts the most of the patient outcome factors including pain, range of motion and ability to perform routine activities. Our case series of olecranon fractures resulted mean MEPS of 89.3 with $57.1 \%$ excellent and $42.9 \%$ good results, and all patients returned to pre-injury daily activities. In cases of Monteggia fracture mean MEPS was 93.8 with $88.9 \%$ excellent result and $11.1 \%$ good results. These results are comparable to studies done by Kloen et al, Niglis et al, Siebenlist et al and Li et al. ${ }^{15-18}$

Various studies reported variety of complications like infection, delayed union, nonunion, implant failure, ulnar nerve dysfunction in various percentages. In our study we observed delayed union in $20 \%(\mathrm{n}=6)$ cases while Yang et al reported $11 \%$ cases of delayed union. ${ }^{19}$

We had some limitations in our study including a small number of patients with subsequent decrease power of study and short duration of follow-up.
Nevertheless, the preliminary good or excellent functional results, the high union rate and low incidence of implant related complications encouraged us to use plating in cases of fracture proximal ulna.

\section{Conclusion}

Comminuted fracture of proximal ulna are challenging, they functionally affect both elbow and the forearm. Fracture morphology and primary elbow instability are the most important prognostic factors for the elbow function. Restoration of ulnohumeral articular congruity and the longitudinal axis of ulna with stable and strong internal fixation are essential to allow early mobilization and prevention of stiffness and ulnohumeral arthritis. The stability of locking construct by providing extra purchase due to shape of plate as well as minimal periosteal compromise, provides high union rates even in osteopenic and comminuted fractures. In our study the management of proximal ulna fracture with locking plate fixation along with early mobilisation, resulted in predictably good union rates and excellent results in terms of patient outcome. Our results are comparable to those reported previously. The good radiological outcomes highlight the usefulness of locking plate osteosynthesis in cases of proximal ulna fractures, provided optimal plate positioning achieved.

\section{References}

1. Hak DJ, and Golladay GJ. Olecranon fractures: treatment options. J Am Acad Orthop Surg. 2000;8:266-275.

2. Quintero J, Vareck T. Olecranon, radial head, and complex elbow injuries. In: Ruedi TP, Buckley RE, Moran CG, eds. AO principles of fracture management, vol 2: Specific fractures, 2nd edn. Stuttgart: Thieme, 2007:626-41.

3. Newman SDS, Mauffrey C, and Krikler S. Olecranon fractures. Injury. 2009;40:575-581.

4. Sahajpal D, and Wright TW. Proximal ulna fractures. $J$ Hand Surg Am. 2009;34:357-362.

5. Nork SE, Jones CB, Henley MB. Surgical treatment of olecranon fractures. Am J Orthop. 2001;30:577-586.

6. Ring D, Tavakolian J, Kloen P. Loss of alignment after surgical treatment of posterior Monteggia fractures: salvage with dorsal contoured plating. J Hand Surg Am. 2004;29:694-702.

7. Fyfe IS, Mossad MM, and Holdsworth BJ. Methods of fixation of olecranon fractures. An experimental mechanical study. J Bone Joint Surg Br. 1985;67:367372.

8. Gordon MJ, Budoff JE, Yeh ML. Comminuted olecranon fractures: a comparison of plating methods. J Shoulder Elbow Surg. 2006;21:386-393.

9. Waddell G, and Howat TW. A technique of plating severe olecranon fractures. Injury. 1973;5:135-140.

10. Lucke M. Olecranonfrakturen. In Stöckle U. (eds): Ellenbogenchirurgie. München: Elsevier, 2009;94-103.

11. Tan SL, and Balogh ZJ. Inidications and limitations of locked plating. Injury. 2009;40:683-691.

12. Jupiter JB, Leibvic SJ, Ribbans W. The posterior Monteggia lesion. J Orthop Trauma. 1991;54:395-402.

13. Bado JL. The Monteggialesion.ClinOrthop.1967;50:7186. 
14. Wang YH, Tao R, Xu H, Cao Y, Zhou ZY, Xu SZ. Midterm outcomes of contoured plating for comminuted fractures of the olecranon. Orthop Surg. 2011;3(3):17680.

15. Siebenlist S, Torsiglieri T, Kraus T, Burghardt RD, Stöckle U et al. Comminuted fractures of the proximal ulna-Preliminary results with ananatomically preshaped locking compression plate (LCP) system. Injury. 2010;41(12):1306-11.

16. Niglis L, Bonnomet F, Schenck B, Brinkert D, Marco A $D$ et al. Critical analysis of olecranon fracture management by pre-contoured locking plates, Orthopaedics \&Traumatology: Surgery \& Research. 2015;101(2):201-7.

17. Kloen P, Buijze GA. Treatment of proximal ulna and olecranon fractures by dorsal plating. Oper Orthop Traumatol. 2009;21(6):571-85.
18. Li T, Jiang XY, Zhang J, Cao QY, Zhang LD, Liu XH, et al. Posterior monteggia fracture-dislocations of proximal ulna. Zhonghua Wai K ZaZhi. 2009;47(12):899-902.

19. Yang M, Zhang DY, Fu ZG et al. Report of 11 cases of the comminuted olecranon fracture treatment with anatomically preshaped locking compression plate (LCP). Journal of Peking University (Health sciences). 2011;43(5):671-4.

How to cite this article: Singhal S K, Sahni V K, Garg M, Gupta A. Outcome of locking plate fixation in cases of comminuted proximal ulna fractures. Indian J Orthop Surg. 2018;4(4):332-337. 